\title{
Perioperative Implementation of Continuous Positive Airway Pressure: A Review of the Considerations
}

\author{
Suzanne Karan*, Shira Black and Falan Mouton
}

Department of Anesthesiology, University of Rochester, Rochester, NY, USA

\begin{abstract}
OSA patients present unique challenges in the peri-operative period. They routinely require more monitoring, oxygen therapy, unplanned ICU admissions, longer hospital stays, and have more adverse events than healthy counterparts. Some data suggest that perioperative CPAP use is associated with reduced morbidity and mortality of patients with OSA, and yet its application remains inconsistent. This review aims to summarize existing literature on the peri-operative use of CPAP, identify barriers to its implementation, and begin defining an algorithm for the practical application of peri-operative CPAP.
\end{abstract}

Keywords: CPAP, obstructive sleep apnea, perioperative.

\section{INTRODUCTION}

Obstructive sleep apnea (OSA) remains an underappreciated cause of morbidity and mortality in the general population. The syndrome affects $2 \%-9 \%$ of women and $4 \%-24 \%$ of men [1] with $80 \%$ or more of the population as yet undiagnosed [2]. OSA occurs when a compromised airway collapses, disrupting the flow of air during sleep. With the ensuing hypoxia and hypercarbia, the respiratory drive is stimulated, a stronger inspiratory effort is made, and the cycle repeats itself throughout the night. The gold standard for diagnosis of OSA remains the polysommnogram(PSG). During the sleep study a patient must be observed to have at least five or more apneas or hypopneas per hour of sleep [3] which last at least 10 seconds each. Severe OSA is generally defined as 30 or more apneic and hypopneic events per hour [4].

Post-operative changes amplify the disease [5-9]. Residual anesthetics weaken the muscles of the upper airway [10, $11]$ and depress the respiratory drive $[12,13]$. Thus, a mild pre-operative case of OSA can easily become a severe postoperative one. OSA patients require more monitoring, oxygen therapy, and unplanned ICU admissions [14]. When compared with controls, OSA patients have longer hospital stays and more adverse events than non-OSA patients [15]. Additionally, an OSA diagnosis is associated with increased post-operative complications, including, but not limited to: airway obstruction, cardiac arrhythmias, hypoxemia, encephalopathy and death $[14,16]$. Adverse outcomes related to respiratory events remains the largest class of injuries reported in the American Society of Anesthesiology Closed Claims study - in cases involving general anesthesia, sedation and monitored anesthetic care (MAC) [17].

It is clear from the medical literature that continuous positive airway pressure (CPAP) immediately reverses sleep

*Address correspondence to this author at the Department of Anesthesiology, University of Rochester, Rochester, NY; Tel: 585-275-1384;

Fax: 585-276-0122; E-mail: Suzanne_Karan@URMC.Rochester.edu related apnea and hypopnea by acting as a pneumatic splint $[18,19]$; though, the benefit of CPAP during the postoperative period, remains the subject of debate [20]. While some studies have suggested a role for CPAP for diagnosed and suspected OSA patients post-operatively, there is still a paucity of evidence on which to develop a protocol for its standardized utility.

Though the benefit of CPAP would seem intuitive, its application remains the subject of debate [20]. How do we identify the undiagnosed OSA patient? Is it necessary for every patient with a diagnosis of OSA to receive CPAP, or can it be tailored to the severity of the disease and the procedure. Should CPAP be applied preoperatively, postoperatively, or both? If a patient has a diagnosis of OSA but does not use CPAP at home should it mandatorily be applied perioperatively? This review paper aims to summarize the existing literature on the perioperative use of CPAP and to start defining an algorithm for its practical application. Additionally, it aims to address gaps in our knowledge and practice in order to direct further research.

\section{POST OP CPAP REDUCES COMPLICATIONS}

It has been shown in several studies that OSA patients have a greater incidence of postoperative complications [15, $21,22]$. However, the postoperative application of CPAP has not been proven to be curative of these problems in a randomized double-blinded trial. In an unblinded study by Squadrone et al., CPAP application to treat hypoxemia after major abdominal surgery significantly reduced re-intubation rates, and correlated with a reduction in ICU length of stay, postoperative pneumonia, infection, sepsis, and death. Another review of 16 cases [23] reported a reduction in postoperative complications in patients who used CPAP therapy preoperatively, upon extubation, and nearly continuously for 24 to 48 hours after surgery. While supplemental oxygen alone may prove beneficial for many patients with hypoxemia, its use should be cautioned in the surgical OSA patient population, as it may reduce the hypoxic respiratory drive, 
thereby increasing the incidence and duration of apneic episodes [24].

\section{THE SEVERITY OF OSA MAY PREDICT THE POSTOPERATIVE COURSE}

Though presence of signs and symptoms of OSA are generally elicited during the preoperative interview, it is unknown whether the severity of the disease predicts a worse outcome. If a patient's home CPAP setting is $12 \mathrm{~cm} \mathrm{H}_{2} \mathrm{O}$, is he/she worse off than one whose setting is only $5 \mathrm{~cm} \mathrm{H}_{2} \mathrm{O}$ ? Or, are both of these patients better off than someone who needs CPAP but does not use it?

Schwartz and colleagues identified a means to predict the potential for upper airway collapse by capitalizing on the reverse of CPAP - namely, by using negative airway pressure to measure the collapsibility of the upper airway [25]. In short, the theory is based upon the modeling of the upper airway after a Starling resistor; being a collapsible tube between two non-collapsible segments. With this reasoning, if positive airway pressure splints open the upper airway in a dose dependent fashion, then it can be theorized that negative airway pressure application potentiates the collapse of the upper airway in a dose dependent fashion. The level of negative airway pressure beyond which airway collapse occurs is called the critical pharyngeal pressure (Pcrit). The value of the Pcrit has been validated and reproduced across many research studies as a predictor of airway compromise that varies by patient factors [26], drugs [13, 27-30], and position of head and body [31]. This value has yet to be validated in the clinical setting, but Eastwood and colleagues correlated the value of the Pcrit to the severity of AHI in a small cohort [32]. Though it remains unknown whether the absolute AHI or level of CPAP used at home predicts worse outcomes perioperatively, the measure of Pcrit holds promise as a laboratory method to tease out the variables that interact in causing more vulnerability to the upper airway.

Another measure of severity of OSA that has predicted outcomes is the oxygen desaturation index (ODI). A change of $4 \%$ from baseline (ODI- $4 \%$ ) has been identified in the literature as differentiating patients who have worse postoperative outcomes. In a study by Hwang et al., patients with ODI- $4 \%$ of five or more times per hour (ODI4\% $\geq 5$ ), had significantly greater incidences for complications than those with ODI $4 \%<5$. Patients with ODI $4 \% \geq 5$ had prolonged PACU stays as well [22].

\section{HOW DO WE DETERMINE WHEN TO APPLY POST- OPERATIVE CPAP AND IN WHOM?}

Even without a firm evidence base, experts still recommend postoperative application of CPAP in select patient groups [33]. It seems obvious that those who use CPAP at home would make up the largest group of postoperative CPAP users, and then additional patient populations would be identified to benefit from this treatment. But, even in home CPAP users there remain questions regarding titration of pressure and duration of use to confer the greatest perioperative benefit. Thus, we are left with many more questions than answers when considering the determination of CPAP use post-operatively.

\section{THERE ARE MANY STRATIFICATIONS OF OSA PATIENTS}

In considering the selection of patients for CPAP use postoperatively, we will break down the following sections by:

1 Home users of CPAP,

2 Non-compliant patients, and

3 Suspected OSA patients who have never been prescribed CPAP.

Contraindication to CPAP use postoperatively will also be touched upon. Finally, we considered a separate indication for CPAP use during ambulatory cases that require sedation.

\section{Home CPAP Users}

The 2006 ASA guidelines regarding the perioperative care of OSA patients state that patients who use CPAP at night should use CPAP postoperatively. It also states that OSA patients should receive monitoring in additional duration and frequency postoperatively. While the latter recommendation seems vague in establishing timing for the care of these patients, it would seem that the former recommendation is straightforward. As we start to devise a protocol aimed at implementing the use of CPAP appropriately in the postoperative period, it would seem that the easiest population to implement post-op CPAP use within is precisely in those who use CPAP at home.

One study [34] demonstrated that when patients were screened for home CPAP/BiPAP use, previous positive test for OSA, snoring, or daytime sleepiness, and evaluated postoperatively for respiratory compromise by a respiratory therapist in the PACU and placed on CPAP when necessary, the hospital eliminated all cases of acute respiratory compromise during the treatment interval. It may also seem obvious that those patients who use CPAP at home would naturally be expected to wear this device while in the hospital and in bed. In a study by Ramachandran et al., $83 \%$ of patients who used CPAP at home brought their machines to the hospital for use. However, another study by Liao et al., showed only $49 \%$ of OSA patients to actually receive CPAP postoperatively [14]. In order to encourage perioperative use of CPAP amongst home using patients, some hospitals have implemented a service that checks CPAP machines preoperatively, and patients are called to bring in their own machines and masks.

It should be noted that even when CPAP machines are brought in from home for hospital use, the settings may still need to be adjusted to account for the increased collapsibility of the airways. For example, one study showed a lack of CPAP efficacy in the first 24 hours postoperatively when patients used their CPAP at the polysomnographicprescribed pressure settings [16]. However, this finding may have been the result of an inadequate postoperative CPAP titration, as narcotics and anesthesia decrease upper airway tone, and their administration may necessitate increased levels of CPAP in the postoperative period. This consideration was taken into effect by Ramachandran et al., as they im- 
plemented the use of a respiratory therapist who was charged with titrating CPAP level to effect in their study [34].

\section{Patients Who Do Not Use Their Prescribed CPAP}

Most patients who have been prescribed home CPAP are somewhat non-compliant with this therapy [35]. Some studies cite $50 \%$ to $80 \%$ of OSA patients who are prescribed to use CPAP actually using their device [36, 37]. And while Liao et al., reported that $63 \%$ of patients who presented for elective surgical procedures were compliant with their CPAP [14], the average duration of use is generally 3.4 to 4.5 hours per night [38]. Of 65 diagnosed OSA patients, Gupta reported only 33 to use CPAP preoperatively [15]. Patient CPAP compliance is generally hindered by the obtrusive nature of the device [19].

Currently, we lack a robust evidence base to support the prescription of CPAP in the hospital for all patients who are non-compliant with its use at home. One can certainly argue that patients who have been prescribed CPAP, but do not use it, are more at risk than those who do use it. The risks involved include suboptimization of sleep and the chronic toll of nighttime hypoxemia in causing cardiac side effects such as arrhythmias and hypertension, to include a few. And while it seems reasonable to prescribe CPAP for those patients who need it in preparation for elective surgery, we still do not have an evidence base to guide the acceptable duration of treatment. On the flip side, these patients would probably also benefit from postoperative application of CPAP for select cases; though, we do not have the evidence, either, to support its standard use. Hospitals that are considering implementing protocols for CPAP application postoperatively should take into account the availability of the resources to titrate the appropriate level of CPAP and the follow up of these patients after discharge with the pulmonary medicine service. In our institution, any patient who receives an order for the "new" application of CPAP needs to also receive a consult from the critical care service in order to ensure continuity of post-operative care.

\section{The Suspected OSA Patient}

The fact remains, though, that OSA is notoriously under diagnosed. In the perioperative setting, Bolden identified 438 of a total of 22,067 (2\%) surgical patients to have known or suspected OSA [16]. Recognizing patients with OSA is critical in determining who could potentially benefit from CPAP. In the literature, patients suspected to have OSA (identified because they report a history of snoring among other symptoms) are often lumped into the moderate-severe OSA patient category with fairly reasonable correlation as described by Young and colleagues [39]. However, the literature varies in its definition of the "suspected OSA" group and this makes the review of treatment effects difficult to interpret. Chung and colleagues have established well validated screening methods for standardizing the identification of the suspected OSA group. If these patients are properly identified preoperatively, the need for postoperative CPAP would be more easily implemented. Unfortunately, most of these patients are still being identified during postoperative quality improvement review of a poor outcome. Once identified, this group of patients should be treated similarly to those who have been prescribed CPAP but do not use it.

\section{THE ROLE FOR CPAP DURING SEDATION REMAINS POORLY DEFINED}

The majority of sedations in the United States are overseen by non-Anesthesiologists, such as Gastroenterologists and Radiologists and administered by nurses. There are many reports of the safe administration of these sedations, even with drugs not specifically approved for this indication, such as propofol $[40,41]$. However, the majority of the morbidity and mortality related to sedation practice is the result of respiratory compromise. There is a substantial body of evidence that supports the notion that the respiratory vulnerability does not only lie in the central control of respiration (cortical drive), but actually moreover in the maintenance of a patent upper airway. Upper airway collapse has been reliably evidenced in the laboratory during moderate sedation even in healthy non-OSA subjects $[12,42]$. Thus, OSA patients who already collapse their airways during "normal" sleep would likely be at increased risk of heightened airway collapsibility when normal sleep interacts with sedation/narcosis. In the gastrointestinal endoscopy literature, OSA has been diagnosed in patients who have been noted to snore during their sedation for outpatient colonscopy [43, 44]. Some have advocated for the application of CPAP during preoxygenation and as an adjunct to sedation during regional anesthesia [45]. During induction of anesthesia, for example, preoxygenation with $100 \%$ oxygen until the exhaled or end-tidal oxygen is at least $90 \%$ can be accomplished by using CPAP at $10 \mathrm{~cm} \mathrm{H}_{2} \mathrm{O}$ for 3 to 5 min with the patient in a $25^{\circ}$ head-up position [46]. This clinical management pearl is applicable to many at-risk patients presenting for sedation (mostly performed by non-anesthesiologists) who would benefit from some degree of upper airway splinting with proper positioning and not just by the application of oxygenation. Others have even advocated the use of a jaw thrust device [47] and other airway maintenance maneuvers (including CPAP) during upper endoscopies in order to maintain airway patency [48]. Future studies to validate the effectiveness of CPAP to ensure safer sedation practices are needed.

\section{POST OP CPAP IS NOT WITHOUT RISK}

While it seems like we made the argument for everyone being on CPAP who has a diagnosis or suspicion for OSA, there are reported contraindications and hesitations for its use in the immediate postoperative period. For instance, CPAP has not been universally accepted for patients following upper gastrointestinal surgery because of concerns that pressurized air will inflate the stomach and proximal intestine, resulting in anastomotic disruption and postoperative nausea and vomiting [49]. Others, however, have recommended its use specifically for these cases [50] with good evidence of it not increasing the risk of developing postoperative anastomotic leaks [51]. There has yet to be a general consensus for its benefit outweighing the hypothesized risks. Recommended practice would be to consult with the surgeon before implementing CPAP postoperatively so the hypothetical risks and benefits may be weighed by the entire health care team on a case by case basis. If CPAP is indeed contraindicated (i.e specific facial surgeries), these patients must be monitored closely in the appropriate setting and may require prolonged intubation, as well. 


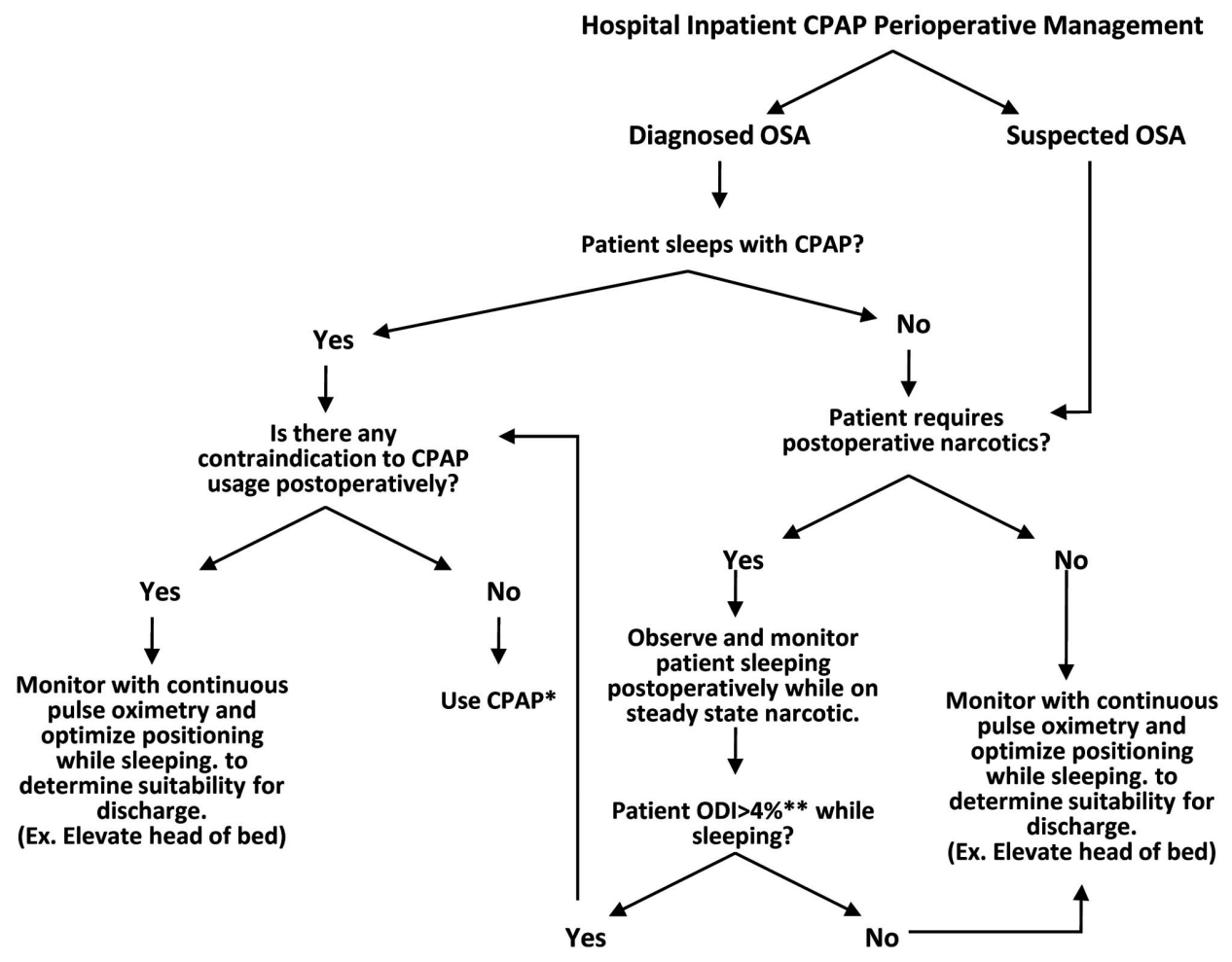

-Settings should be adjusted by a respiratory therapist to optimize ventilation. $* *$ ODI $4 \%$ : oxygen desaturation index greater than four percent.

Fig. (1). This algorithm is meant to guide the management for application of post-operative CPAP. Although its efficacy has not been proven in randomized controlled trials, it remains a logical approach for the surgical patient based on the review of the literature cited in the present article. * Settings may be adjusted by a respiratory therapist to optimize ventilation.

$* * \mathrm{ODI}>4 \%$ : oxygen desaturation index greater than four percent. $\infty$ Contraindications include, but are not limited to, select gastrointestinal procedures involving anastomoses, facial surgeries where a CPAP mask would not be tolerated, and cases where the surgeon indicates its use to be problematic.

Taking this all into account, the following is an algorithm (Fig. 1) for determining CPAP use postoperatively. Stratification of CPAP use by postoperative need for narcotic medication was considered as per the ASA guidelines concerning the perioperative care of OSA patients [52].

\section{REFERENCE}

[1] Young T, Evans L, Finn L, Palta M. Estimation of the clinically diagnosed proportion of sleep apnea syndrome in middle-aged men and women. Sleep 1997; 20: 705-6.

[2] Stierer TL, Wright C, George A, Thompson RE, Wu CL, Collop N. Risk assessment of obstructive sleep apnea in a population of patients undergoing ambulatory surgery. J Clin Sleep Med 2010; 6: 467-72.

[3] Gould GA, Whyte KF, Rhind GB, et al. The sleep hypopnea syndrome. Am Rev Respir Dis 1988; 137: 895-8.

[4] The Report of an American Academy of Sleep Medicine Task Force. Sleep-related breathing disorders in adults: recommendations for syndrome definition and measurement techniques in clinical research. Sleep 1999; 22: 667-89.

[5] Benumof JL. Obstructive sleep apnea in the adult obese patient: implications for airway management. Anesthesiol Clin North Am 2002; 20: 789-811.

[6] Marshall BE, Wyche MQ, Jr. Hypoxemia during and after anesthesia. Anesthesiology 1972; 37: 178-209.

[7] Catley DM, Thornton C, Jordan C, Lehane JR, Royston D, Jones JG. Pronounced, episodic oxygen desaturation in the postoperative period: its association with ventilatory pattern and analgesic regimen. Anesthesiology 1985; 63: 20-8.

[8] Hines R, Barash PG, Watrous G, O'Connor T. Complications occurring in the postanesthesia care unit: a survey. Anesth Analg 1992; 74: 503-9.
[9] Hillman DR, Loadsman JA, Platt PR, Eastwood PR. Obstructive sleep apnoea and anaesthesia. Sleep Med Rev 2004; 8: 459-71.

[10] Hillman DR, Walsh JH, Maddison KJ, et al. Evolution of changes in upper airway collapsibility during slow induction of anesthesia with propofol. Anesthesiology 2009; 111: 63-71.

[11] Eikermann M, Fassbender P, Zaremba S, et al. Pentobarbital dosedependently increases respiratory genioglossus muscle activity while impairing diaphragmatic function in anesthetized rats. Anesthesiology 2009; 110: 1327-34.

[12] Bailey PL, Pace NL, Ashburn MA, Moll JW, East KA, Stanley TH Frequent hypoxemia and apnea after sedation with midazolam and fentanyl. Anesthesiology 1990; 73: 826-30.

[13] Norton JR, Karan SB, Bailey PL, Voter W, Palmer LS, Ward DS. The effects and interaction of sedative doses of intravenous midazolam or propofol, and hypoxia, on upper airway obstruction. Anesthesiolgy (abstract). ASA. Ref Type: Abstract 2003.

[14] Liao P, Yegneswaran B, Vairavanathan S, Zilberman P, Chung F. Postoperative complications in patients with obstructive sleep apnea: a retrospective matched cohort study. Can J Anaesth 2009; 56: 819-28.

[15] Gupta RM, Parvizi J, Hanssen AD, Gay PC. Postoperative complications in patients with obstructive sleep apnea syndrome undergoing hip or knee replacement: a case-control study. Mayo Clin Proc 2001; 76: 897-905

[16] Bolden N, Smith CE, Auckley D, Makarski J, Avula R. Perioperative complications during use of an obstructive sleep apnea protocol following surgery and anesthesia. Anesth Analg 2007; 105: 1869-70.

[17] Bhananker SM, Posner KL, Cheney FW, Caplan RA, Lee LA, Domino KB. Injury and liability associated with monitored anesthesia care: a closed claims analysis. Anesthesiology 2006; 104: 228-34. 
[18] Sullivan CE, Issa FG, Berthon-Jones M, Eves L. Reversal of obstructive sleep apnoea by continuous positive airway pressure applied through the nares. Lancet 1981; 1: 862-5.

[19] Flemons WW. Clinical practice. Obstructive sleep apnea. N Engl J Med 2002; 347: 498-504.

[20] Drummond GB, Stedul K, Kingshott R, et al. Automatic CPAP compared with conventional treatment for episodic hypoxemia and sleep disturbance after major abdominal surgery. Anesthesiology 2002; 96: 817-26.

[21] Riley RW, Powell NB, Guilleminault C, Pelayo R, Troell RJ, Li KK. Obstructive sleep apnea surgery: risk management and complications. Otolaryngol Head Neck Surg 1997; 117: 648-52.

[22] Hwang D, Shakir N, Limann B, et al. Association of sleepdisordered breathing with postoperative complications. Chest 2008; 133: 1128-34.

[23] Rennotte MT, Baele P, Aubert G, Rodenstein DO. Nasal continuous positive airway pressure in the perioperative management of patients with obstructive sleep apnea submitted to surgery. Chest 1995; 107: 367-74.

[24] Fletcher EC, Munafo DA. Role of nocturnal oxygen therapy in obstructive sleep apnea. When should it be used? Chest 1990; 98: 1497-504.

[25] Schwartz AR, Smith PL, Wise RA, Gold AR, Permutt S. Induction of upper airway occlusion in sleeping individuals with subatmospheric nasal pressure. J Appl Physiol 1988; 64: 535-42.

[26] Gleadhill IC, Schwartz AR, Schubert N, Wise RA, Permutt S, Smith PL. Upper airway collapsibility in snorers and in patients with obstructive hypopnea and apnes. Am Rev Respir Dis 1991; 143: 1300-3.

[27] Litman RS, McDonough JM, Marcus CL, Schwartz AR, Ward DS. Upper airway collapsibility in anesthetized children. Anesth Analg 2006; 102: 750-4.

[28] Litman RS, Hayes JL, Basco MG, Schwartz AR, Bailey PL, Ward DS. Use of dynamic negative airway pressure (DNAP) to assess sedative-induced upper airway obstruction. Anesthesiology 2002; 96: 342-5.

[29] Eastwood PR, Szollosi I, Platt PR, Hillman DR. Collapsibility of the upper airway during anesthesia with isoflurane. Anesthesiology 2002; 97: 786-93.

[30] Eastwood PR, Platt PR, Shepherd K, Maddison K, Hillman DR. Collapsibility of the upper airway at different concentrations of propofol anesthesia. Anesthesiology 2005; 103: 470-7.

[31] Isono S, Tanaka A, Nishino T. Lateral position decreases collapsibility of the passive pharynx in patients with obstructive sleep apnea. Anesthesiology 2002; 97: 780-5.

[32] Eastwood PR, Szollosi I, Platt PR, Hillman DR. Comparison of upper airway collapse during general anaesthesia and sleep. Lancet 2002; 359: 1207-9.

[33] A Report by the American Society of Anesthesiologists Task Force on Sedation and Analgesia by Non-Anesthesiologists. Practice guidelines for sedation and analgesia by non-anesthesiologists. Anesthesiology 1996; 84: 459-71.

[34] Ramachandran SK, Kheterpal S, Haas CF, Saran KA, Tremper KK. Automated notification of suspected obstructive sleep apnea patients to the perioperative respiratory therapist: a pilot study. Respir Care 2010; 55: 414-8.

[35] Lindberg E, Berne C, Elmasry A, Hedner J, Janson C. CPAP treatment of a population-based sample--what are the benefits and the treatment compliance? Sleep Med 2006; 7: 553-60.
[36] American Thoracic Society. Indications and standards for use of nasal continuous positive airway pressure (CPAP) in sleep apnea syndromes. Official statement adopted March 1944. Am J Respir Crit Care Med 1994; 150: 1738-45.

[37] McArdle N, Devereux G, Heidarnejad H, Engleman HM, Mackay TW, Douglas NJ. Long-term use of CPAP therapy for sleep apnea/hypopnea syndrome. Am J Respir Crit Care Med 1999; 159: $1108-14$.

[38] Weaver TE, Maislin G, Dinges DF, et al. Relationship between hours of CPAP use and achieving normal levels of sleepiness and daily functioning. Sleep 2007; 30: 711-9.

[39] Young T, Palta M, Dempsey J, Skatrud J, Weber S, Badr S. The occurrence of sleep-disordered breathing among middle-aged adults. N Engl J Med 1993; 328: 1230-5.

[40] Bitar G, Mullis W, Jacobs W, et al. Safety and efficacy of officebased surgery with monitored anesthesia care/sedation in 4778 consecutive plastic surgery procedures. Plast Reconstr Surg 2003; 111: 150-6.

[41] Walker JA, McIntyre RD, Schleinitz PF, et al. Nurse-administered propofol sedation without anesthesia specialists in 9152 endoscopic cases in an ambulatory surgery center. Am J Gastroenterol 2003; 98: 1744-50.

[42] Norton JR, Ward DS, Karan S, et al. Differences between midazolam and propofol sedation on upper airway collapsibility using dynamic negative airway pressure. Anesthesiology 2006; 104: 115564.

[43] Sharma VK, Galli W, Haber A, et al. Unexpected risks during administration of conscious sedation: previously undiagnosed obstructive sleep apnea. Ann Intern Med 2003; 139: 707-8.

[44] Sharara AI, El Zahabi L, Maasri K, et al. Persistent snoring under conscious sedation during colonoscopy is a predictor of obstructive sleep apnea. Gastrointest Endosc 2010; 71: 1224-30.

[45] Nozaki-Taguchi N, Isono S, Nishino T, Numai T, Taguchi N. Upper airway obstruction during midazolam sedation: modification by nasal CPAP. Can J Anaesth 1995; 42: 685-90.

[46] Herriger A, Frascarolo P, Spahn DR, Magnusson L. The effect of positive airway pressure during pre-oxygenation and induction of anaesthesia upon duration of non-hypoxic apnoea. Anaesthesia 2004; 59: 243-7.

[47] Mandel J. Airway appliances in endoscopy. Tech Gastrointest Endosc 2009; 11: 188-91.

[48] Atkins J. Ventilation strategies in gastrointestinal endoscopy. Tech Gastrointest Endosc 2009; 11: 192-6.

[49] Meng L. Postoperative nausea and vomiting with application of postoperative continuous positive airway pressure after laparoscopic gastric bypass. Obes Surg 2010; 20: 876-80.

[50] Oberg B, Poulsen TD. Obesity: an anaesthetic challenge. Acta Anaesth Scand 1996; 40: 191-200.

[51] Verse T. Bariatric surgery for obstructive sleep apnea. Chest 2005; 128: 485-7.

[52] Practice guidelines for the perioperative management of patients with obstructive sleep apnea: a report by the american society of anesthesiologists task force on perioperative management of patients with obstructive sleep apnea. Anesthesiology 2006; 104: 1081-93. 